\title{
Editorial: Health and Performance Assessment in Winter Sports
}

\author{
Jörg Spörri ${ }^{1,2 *}$, Thomas Stögg/ ${ }^{3,4}$ and Kamiar Aminian ${ }^{5}$ \\ ${ }^{1}$ Sports Medical Research Group, Department of Orthopaedics, Balgrist University Hospital, University of Zurich, Zurich, \\ Switzerland, ${ }^{2}$ University Centre for Prevention and Sports Medicine, Department of Orthopaedics, Balgrist University \\ Hospital, University of Zurich, Zurich, Switzerland, ${ }^{3}$ Department of Sport Science and Kinesiology, University of Salzburg, \\ Hallein, Austria, ${ }^{4}$ Red Bull Athlete Performance Centre, Thalgau, Austria, ${ }^{5}$ Laboratory of Movement Analysis and \\ Measurement, Ecole Polytechnique Fédérale de Lausanne, Lausanne, Switzerland
}

Keywords: algorithms, biomechanics, diagnostics, digital health, injury prevention, performance enhancement, sensors, athlete

\section{Editorial on the Research Topic}

\section{Health and Performance Assessment in Winter Sports}

Recent developments in technology and engineering have provided novel solutions for monitoring health and performance, as well as assessing key variables that would not have been easily accessible a few years ago. Options include digital solutions for collecting self-reported data on physical activity, recovery, psychological readiness, or injury (Düking et al., 2018), measurement technologies for quantifying physiological variables (Khundaqji et al., 2020), wearable sensors for motion analysis (Sperlich et al., 2020), and technology-based approaches for performance and load quantification (Lutz et al., 2019). Moreover, customized algorithms and data analytics help to extract and visualize relevant metrics for effective coaching and athletes' health protection (Rommers et al., 2020).

A close relationship between engineers, coaches, sports scientists, and medical professionals ensures the success of healthy sporting activity and the sustainable long-term development of athletes throughout their careers. This is especially true for winter sports and youth athletes. On the one hand, the assessment of health and performance in winter sports is a permanent challenge and, from a technological point of view, particularly difficult. Winter sports take place under extreme and hardly standardisable outdoor conditions. Therefore, various research is done in laboratory situations (e.g., ski simulators, ski ergometers, imitation movements of winter sportsspecific motions, etc.). However, in many cases, there is an evident need to bring the lab to the field and assess health and performance-related aspects within representative real-life settings (Spörri et al., 2016). On the other hand, youth athletes need special care because they are particularly susceptible to the long-term consequences of sports participation and injuries during phases of biological maturation and rapid musculoskeletal growth (Schoeb et al., 2020). However, there is great potential in young athletes being more familiar with digitalization and consequently better able to use new technologies.

Accordingly, the main objective of our Research Topic entitled "Health and Performance Assessment in Winter Sports" was to emphasize the relationship between health, performance, and technology, and to highlight current challenges in the design of innovative measuring systems, wearable sensors, and assessment protocols for examining and monitoring health and performance in sports like Freestyle, Alpine, Nordic and Paralympic skiing. It was also intended to compile research articles focusing on the application of digitalization and

Sports.

Front. Sports Act. Living 3:628574.

doi: 10.3389/fspor.2021.628574

Citation:

Spörri J, Stöggl T and Aminian K (2021) Editorial: Health and Performance Assessment in Winter

OPEN ACCESS

Edited by:

Silvia Fantozzi,

Reviewed by:

Peter A. Federolf,

*Correspondence: Jörg Spörri

Received: 12 November 2020 Accepted: 12 February 2021 Published: 09 March 2021

This article was submitted to Engineering a section of the journal Frontiers in Sports and Active Living technology in the context of performance enhancement, injury prevention, and rehabilitation. 


\section{CURRENT TRENDS OF HEALTH AND PERFORMANCE ASSESSMENT IN WINTER SPORTS}

Recent advances in digitization and measurement technology have also influenced youth and elite winter sports. Current trends of health and performance assessment can be summarized as: (1) new technologies and approaches for motion analysis with a clear tendency toward wearables, large capture volumes and long-term assessments (Supej, 2010; Gilgien et al., 2013, 2015; Fasel et al., 2017a,b; Spörri et al., 2017; Fasel et al., 2018; Gløersen et al., 2018a,b; Takeda et al., 2019; Neuwirth et al., 2020); (2) digital data collections, interdisciplinary/integrative measurement setups, advanced signal processing and state-ofthe-art data analytics such as machine learning in connection with performance assessment and enhancement (Rindal et al., 2017; Jang et al., 2018; Losnegard et al., 2019; Ostrek et al., 2019; Skattebo et al., 2019; Heinrich et al., 2020; Solli et al., 2020); (3) mobile Health (mHealth) applications, supporting engineering solutions, and technology-based athlete screening in the context of injury/illness surveillance and prevention (Steenstrup et al., 2018; Schindelwig et al., 2019; Ellenberger et al., 2020a,b; Franchi et al., 2020; Fröhlich et al., 2020; Hermann and Senner, 2020); and (4) comprehensive monitoring/test protocols, and the objectification of clinical criteria in the return-to-sport context (Jordan et al., 2015a,b; Jordan et al., 2018; Csapo et al., 2019).

Fully in line with these current trends, our Research Topic on "Health and Performance Assessment in Winter Sports" compromises a total of 13 research articles, all of which can be assigned to one of the trend areas mentioned above (see Table 1). Of these articles, 11 were original research articles, one a perspective and one a methods article. Most articles were crosssectional observations, while there were only three articles based on other study designs (one case study, one intervention study, and one literature review).

With respect to new technologies and approaches, Martinez, Brunauer et al. developed and validated a gyroscope-based ski turn detection algorithm for an on-snow application under various conditions. For many health and performancerelated applications in alpine skiing, an exact determination of separate turn cycles is key (Spörri et al., 2012, 2018). An alternative, vertical ground-reaction force(GRF)-based approach was investigated in Martinez, Nakazato et al. In their study, they compared different functional definition criteria, as well as portable force platforms vs. pressure insoles for the determination of turn switches during alpine skiing. Supej et al. summarized the current state of scientific knowledge on methodological and practical aspects of the assessment of alpine skiing performance by Global Navigation Satellite Systems (GNSS) and stimulated future perspectives on the topic. GNSS-based performance assessments have been widely used in research on alpine and cross-country skiers (Supej, 2010; Gilgien et al., 2013, 2015; Gløersen et al., 2018a), and many skiing teams already employ GNSS technology in their daily training and equipment testing routines. Thus, in combination with advanced computer software, they are a good example of how the digital revolution has also taken hold in winter sports.

With regard to performance assessment and enhancement, the current Research Topic offers a broad spectrum of digitalization and technology-driven sample applications in the fields of alpine skiing, cross-country skiing, and biathlon. Alhammoud et al. and Bruhin et al. for example, have used state-ofthe-art wearable measurement technologies to analyse the racecourse (e.g., course setting, steepness, etc.) and motion patterns/performance characteristics (e.g., joint angles, speed, time per turn, turn phases, etc.) of competitive alpine skiers at all levels and within different alpine disciplines. Moreover, Reid et al. used a complex video-based 3D kinematic analysis to quantify the ski motion characteristics during alpine slalom skiing and compare these measures with theoretical predictions based on ski geometry. Two interesting application examples of integrative measurement setups that use combined interdisciplinary research approaches (such as biomechanics and physiology), are the study on the influence of sitting posture on the sit-skiing economy by Lajunen et al. and the study on the determinants of biathlon competition performance by Luchsinger et al. Finally, Steidl-Müller et al. investigated whether the relative age effect in youth competitive alpine skiing changed over the last decade; a comprehensive analysis with the data of more than 1,400 athletes, which benefits from today's digitalization in competitive sports.

In the context of injury/illness surveillance and prevention, the study by Doyle-Baker and Emery presented some interesting new data on physical activity, injury, and illness among adolescent skiers. Despite all technological advances and booming mHealth applications, trustworthy information provided by athletes and self-reported data from various stakeholders of the sport remains an important pillar for load management and injury registration. This also applies to the study by Westin et al. who evaluated a sports-specific anterior cruciate ligament $(\mathrm{ACL})$ injury prevention programme based on a systematic injury monitoring system, installed at Swedish ski high schools. In their study, they reported a promising $45 \%$ reduction in the incidence rate of ACL injuries among young competitive alpine skiers when a prevention program consisting of neuromuscular exercise was implemented. Additionally, the review by Hanstock et al. aimed to provide an overview of pathophysiological responses to exercise at sub-zero temperatures and identify the potential of heat-andmoisture-exchanging breathing devices to prevent airway pathophysiological responses to cold air exercise.

In terms of return-to-sport, this Research Topic is complemented by the case study of Jordan et al. which presented a detailed analytical framework for return-to-sport training and neuromuscular testing used in an elite female alpine ski racer following ACL reconstruction. Interestingly, the authors reported that functional and strength deficits persisted up to 18 months post-surgery; a fact that further supports the use of an athlete monitoring approach that tracks them throughout the return-tosport/return-to-performance transitions, rather than a discrete timepoint clearance approach. In this context, technology has 
TABLE 1 | Overview of the published research articles categorized by area, winter sports, article type/study design, and subjects.

\begin{tabular}{|c|c|c|c|c|}
\hline Area & Winter sports & Article type/Study design & Subjects & References \\
\hline \multicolumn{5}{|l|}{ New technologies and approaches } \\
\hline Gyroscopes for turn switch detection & Alpine skiing & $\begin{array}{l}\text { Original research } \\
\text { article/cross-sectional study }\end{array}$ & $n=11$ & Martinez, Brunauer et al. \\
\hline $\begin{array}{l}\text { Pressure insoles vs. force plates for turn } \\
\text { switch detection }\end{array}$ & Alpine skiing & $\begin{array}{l}\text { Original research/cross-sectional } \\
\text { study }\end{array}$ & $n=20$ & Martinez, Nakazato et al. \\
\hline $\begin{array}{l}\text { GNSS for performance analysis (skier } \\
\text { kinematics) }\end{array}$ & Alpine skiing & $\begin{array}{l}\text { Perspective article/literature } \\
\text { review }\end{array}$ & $\mathrm{n} / \mathrm{a}$ & Supej et al. \\
\hline \multicolumn{5}{|l|}{ Performance assessment and enhancement } \\
\hline $\begin{array}{l}\text { Discipline-dependent knee and hip joint } \\
\text { kinematics using electro-goniometers }\end{array}$ & Alpine skiing & $\begin{array}{l}\text { Original research } \\
\text { article/cross-sectional study }\end{array}$ & $n=20$ & Alhammoud et al. \\
\hline $\begin{array}{l}\text { Level-, course and terrain-dependent } \\
\text { performance analysis by GNSS }\end{array}$ & Alpine skiing & $\begin{array}{l}\text { Original research } \\
\text { article/cross-sectional study }\end{array}$ & $n=57$ & Bruhin et al. \\
\hline $\begin{array}{l}\text { Effect of sitting posture on sit-skiing economy } \\
\text { based on an interdisciplinary/integrative } \\
\text { measurement set-up }\end{array}$ & $\begin{array}{l}\text { Paralympic } \\
\text { Cross-Country skiing }\end{array}$ & $\begin{array}{l}\text { Original research article/cross } \\
\text { sectional study }\end{array}$ & $n=10$ & Lajunen et al. \\
\hline $\begin{array}{l}\text { Skier technique and tactic analysis by } \\
\text { video-based 3D kinematics }\end{array}$ & Alpine skiing & $\begin{array}{l}\text { Original research } \\
\text { article/cross-sectional study }\end{array}$ & $n=6$ & Reid et al. \\
\hline $\begin{array}{l}\text { Relative age effect in youth competitive alpine } \\
\text { skiers based on a comprehensive database }\end{array}$ & Alpine skiing & $\begin{array}{l}\text { Original research } \\
\text { article/cross-sectional study }\end{array}$ & $n=1,466$ & Steidl-Müller et al. \\
\hline \multicolumn{5}{|l|}{ Injury/illness surveillance and prevention } \\
\hline $\begin{array}{l}\text { Self-reported physical activity, injury, and by } \\
\text { using an online questionnaire }\end{array}$ & Alpine skiing & $\begin{array}{l}\text { Original research } \\
\text { article/cross-sectional study }\end{array}$ & $n=96$ & Doyle-Baker and Emery \\
\hline $\begin{array}{l}\text { Exercise in the cold with special focus on } \\
\text { heat-and-moisture-exchanging breathing } \\
\text { devices }\end{array}$ & Cross-Country skiing & $\begin{array}{l}\text { Original research } \\
\text { article/cross-sectional study }\end{array}$ & $\mathrm{n} / \mathrm{a}$ & Hanstock et al. \\
\hline
\end{tabular}

GNSS, Global Navigation Satellite Systems; 3D, Three-dimensional; ACL, anterior cruciate ligament.

great potential for the development of standardized and reliable neuromuscular assessment protocols and objective criteria.

\section{WHERE TO GO FROM HERE?}

Given these general trends, new research articles, and the current state of scientific knowledge on health and performance assessment in winter sports, there are challenges that need to be addressed in the future.

\section{New Technologies and Approaches in General}

- To be attractive for sports practitioners, new technologies and engineering solutions should be easy to use/calibrate and not hinder athletes with cumbersome multiple sensors, fixations, and connections. Ideally, sensors and electronics should be integrated or simply fixed into the athletes' standard equipment.
- However, industry and end-users must care about the objectivity, validity, and reliability of the technology applied. For effective decision-making in coaching and clinical practice, imprecise or even incorrect information can be worse than no additional information.

\section{Health and Performance Assessment}

- New technologies and approaches should be used for providing evidence, not to confirm single observations and personal beliefs.

- A systematic and longitudinal collection of data is crucial. Here proceeding digitalization and technological development will help.

- Drawing practically relevant conclusions from a wealth of data (information) through data analytics (knowledge) will be one of our greatest challenges in the near future. In this context, the ongoing digitalization and new technological trends may provide significant support. 
- An integrative fusion of different technologies and disciplines (e.g., biomechanics, physiology, psychology) will most likely become the new state-of-the-art in terms of health and performance assessment in winter sports.

\section{REFERENCES}

Csapo, R., Hoser, C., Gföller, P., Raschner, C., and Fink, C. (2019). Fitness, knee function and competition performance in professional alpine skiers after ACL injury. J. Sci. Med. Sport 22, S39-S43. doi: 10.1016/j.jsams.2018.06.014

Düking, P., Achtzehn, S., Holmberg, H.-C., and Sperlich, B. (2018). Integrated framework of load monitoring by a combination of smartphone applications, wearables and point-of-care testing provides feedback that allows individual responsive adjustments to activities of daily living. Sensors 18:1632. doi: $10.3390 / \mathrm{s} 18051632$

Ellenberger, L., Jermann, J., Fröhlich, S., Frey, W. O., Snedeker, J. G., and Spörri, J. (2020a). Biomechanical quantification of deadbug bridging performance in competitive alpine skiers: reliability, reference values, and associations with skiing performance and back overuse complaints. Phys. Ther. Sport 45, 56-62. doi: $10.1016 /$ j.ptsp.2020.05.013

Ellenberger, L., Oberle, F., Lorenzetti, S., Frey, W. O., Snedeker, J. G., and Spörri, J. (2020b). Dynamic knee valgus in competitive alpine skiers: observation from youth to elite and influence of biological maturation. Scand. J. Med. Sci. Sports 30, 1212-1220. doi: 10.1111/sms.13657

Fasel, B., Gilgien, M., Spörri, J., and Aminian, K. (2018). A new training assessment method for alpine ski racing: estimating center of mass trajectory by fusing inertial sensors with periodically available position anchor points. Front. Physiol. 9:1203. doi: 10.3389/fphys.2018.01203

Fasel, B., Spörri, J., Schutz, P., Lorenzetti, S., and Aminian, K. (2017a). An Inertial Sensor-Based Method for Estimating the Athlete's Relative Joint Center Positions and Center of Mass Kinematics in Alpine Ski Racing. Front. Physiol 8:850. doi: 10.3389/fphys.2017.00850

Fasel, B., Spörri, J., Schutz, P., Lorenzetti, S., and Aminian, K. (2017b). Validation of functional calibration and strap-down joint drift correction for computing 3D joint angles of knee, hip, and trunk in alpine skiing. PLOS ONE 12:e0181446. doi: 10.1371/journal.pone.0181446

Franchi, M. V., Fitze, D. P., Hanimann, J., Sarto, F., and Spörri, J. (2020). Panoramic ultrasound vs. MRI for the assessment of hamstrings crosssectional area and volume in a large athletic cohort. Sci. Rep. 10:14144. doi: 10.1038/s41598-020-71123-6

Fröhlich, S., Helbling, M., Fucentese, S. F., Karlen, W., Frey, W. O., and Spörri, J. (2020). Injury risks among elite competitive alpine skiers are underestimated if not registered prospectively, over the entire season and regardless of whether requiring medical attention. Knee Surg. Sports Traumatol. Arthrosc. doi: 10.1007/s00167-020-06110-5. [Epub ahead of print].

Gilgien, M., Spörri, J., Chardonnens, J., Kröll, J., Limpach, P., and Müller, E. (2015). Determination of the centre of mass kinematics in alpine skiing using differential global navigation satellite systems. J. Sports Sci. 33, 960-969. doi: 10.1080/02640414.2014.977934

Gilgien, M., Spörri, J., Chardonnens, J., Kröll, J., and Müller, E. (2013). Determination of external forces in alpine skiing using a differential global navigation satellite system. Sensors 13, 9821-9835. doi: 10.3390/s130809821

Gløersen, Ø., Kocbach, J., and Gilgien, M. (2018a). Tracking performance in endurance racing sports: evaluation of the accuracy offered by three commercial GNSS receivers aimed at the sports market. Front. Physiol. 9:1425. doi: 10.3389/fphys.2018.01425

Gløersen, Ø., Losnegard, T., Malthe-Sørenssen, A., Dysthe, D. K., and Gilgien, M. (2018b). Propulsive power in cross-country skiing: application and limitations of a novel wearable sensor-based method during roller skiing. Front. Physiol. 9:1631. doi: 10.3389/fphys.2018.01631

Heinrich, A., Hansen, D. W., Stoll, O., and Cañal-Bruland, R. (2020). The impact of physiological fatigue and gaze behavior on shooting performance in expert biathletes. J. Sci. Med. Sport 23, 883-890. doi: 10.1016/j.jsams.2020.02.010

\section{AUTHOR CONTRIBUTIONS}

JS, TS, and KA designed and edited this Research Topic. JS wrote the first draft of the corresponding editorial. All authors contributed to the manuscript and approved the final version.

Hermann, A., and Senner, V. (2020). Knee injury prevention in alpine skiing. A technological paradigm shift towards a mechatronic ski binding. J. Sci. Med. Sport. doi: 10.1016/j.jsams.2020.06.009. [Epub ahead of print].

Jang, J., Ankit, A., Kim, J., Jang, Y. J., Kim, H. Y., Kim, J. H., et al. (2018). A unified deep-learning model for classifying the cross-country skiing techniques using wearable gyroscope sensors. Sensors 18:3819. doi: 10.3390/s18113819

Jordan, M. J., Aagaard, P., and Herzog, W. (2015a). Lower limb asymmetry in mechanical muscle function: a comparison between ski racers with and without ACL reconstruction. Scand. J. Med. Sci. Sports 25, e301-e309. doi: $10.1111 /$ sms.12314

Jordan, M. J., Aagaard, P., and Herzog, W. (2015b). Rapid hamstrings/quadriceps strength in ACL-reconstructed elite alpine ski racers. Med. Sci. Sports Exerc. 47, 109-119. doi: 10.1249/MSS.0000000000000375

Jordan, M. J., Aagaard, P., and Herzog, W. (2018). A comparison of lower limb stiffness and mechanical muscle function in ACL-reconstructed, elite, and adolescent alpine ski racers/ski cross athletes. J. Sport Health Sci. 7, 416-424. doi: 10.1016/j.jshs.2018.09.006

Khundaqji, H., Hing, W., Furness, J., and Climstein, M. (2020). Smart shirts for monitoring physiological parameters: scoping review. JMIR MHealth UHealth 8:e18092. doi: 10.2196/18092

Losnegard, T., Tosterud, O. K., Trøen, E., Carlsen, C. H., Paulsen, G., and Rud, B. (2019). The influence of pole lengths on O2-cost, kinematics, and performance in double poling at high speeds before and after a training period with long poles. Eur. J. Appl. Physiol. 119, 2579-2587. doi: 10.1007/s00421-019-04237-z

Lutz, J., Memmert, D., Raabe, D., Dornberger, R., and Donath, L. (2019). Wearables for integrative performance and tactic analyses: opportunities, challenges, and future directions. Int. J. Environ. Res. Public Health 17:59. doi: 10.3390/ijerph17010059

Neuwirth, C., Snyder, C., Kremser, W., Brunauer, R., Holzer, H., and Stöggl, T. (2020). Classification of alpine skiing styles using GNSS and inertial measurement units. Sensors 20:4232. doi: 10.3390/s20154232

Ostrek, M., Rhodin, H., Fua, P., Müller, E., and Spörri, J. (2019). Are existing monocular computer vision-based 3D Motion capture approaches ready for deployment? A methodological study on the example of alpine skiing. Sensors 19:4323. doi: 10.3390/s19194323

Rindal, O., Seeberg, T., Tjønnås, J., Haugnes, P., and Sandbakk, Ø. (2017). Automatic classification of sub-techniques in classical cross-country skiing using a machine learning algorithm on micro-sensor data. Sensors 18:75. doi: 10.3390/s18010075

Rommers, N., Rössler, R., Verhagen, E., Vandecasteele, F., Verstockt, S., Vaeyens, R., et al. (2020). A machine learning approach to assess injury risk in elite youth football players. Med. Sci. Sports Exerc. 52, 1745-1751. doi: 10.1249/MSS.0000000000002305

Schindelwig, K., Platzer, H.-P., Mössner, M., and Nachbauer, W. (2019). Safety recommendations of winter terrain park jumps into airbags. J. Sci. Med. Sport 22, S50-S54. doi: 10.1016/j.jsams.2018.09.229

Schoeb, T., Peterhans, L., Fröhlich, S., Frey, W. O., Gerber, C., and Spörri, J. (2020). Health problems in youth competitive alpine skiing: a 12-month observation of 155 athletes around the growth spurt. Scand. J. Med. Sci. Sports 29, 1254-1264. doi: $10.1111 /$ sms. 13740

Skattebo, Ø., Losnegard, T., and Stadheim, H. K. (2019). Double-poling physiology and kinematics of elite cross-country skiers: specialized longdistance versus all-round skiers. Int. J. Sports Physiol. Perform 14, 1190-1199. doi: 10.1123/ijspp.2018-0471

Solli, G. S., Sandbakk, S. B., Noordhof, D. A., Ihalainen, J. K., and Sandbakk, $\varnothing$. (2020). Changes in self-reported physical fitness, performance, and side effects across the phases of the menstrual cycle among competitive endurance athletes. Int. J. Sports Physiol. Perform 15, 1324-1333. doi: 10.1123/ijspp.2019-0616 
Sperlich, B., Aminian, K., Düking, P., and Holmberg, H.-C. (2020). Editorial: wearable sensor technology for monitoring training load and health in the athletic population. Front. Physiol. 10:1520. doi: 10.3389/fphys.2019. 01520

Spörri, J., Kröll, J., Fasel, B., Aminian, K., and Müller, E. (2017). the use of body worn sensors for detecting the vibrations acting on the lower back in alpine ski racing. Front. Physiol 8:522. doi: 10.3389/fphys.2017.00522

Spörri, J., Kröll, J., Schwameder, H., and Müller, E. (2018). The role of path length- and speed-related factors for the enhancement of section performance in alpine giant slalom. Eur. J. Sport Sci. 18, 911-919. doi: 10.1080/17461391.2018.1453870

Spörri, J., Kröll, J., Schwameder, H., Schiefermüller, C., and Müller, E. (2012). Course setting and selected biomechanical variables related to injury risk in alpine ski racing: an explorative case study. Br. J. Sports Med. 46, 1072-1077. doi: 10.1136/bjsports-2012-091425

Spörri, J., Schiefermüller, C., and Müller, E. (2016). Collecting kinematic data on a ski track with optoelectronic stereophotogrammetry: a methodological study assessing the feasibility of bringing the biomechanics lab to the field. PLOS ONE 11:e0161757. doi: 10.1371/journal.pone.0161757

Steenstrup, S. E., Mok, K. M., Mcintosh, A. S., Bahr, R., and Krosshaug, T. (2018). Head impact velocities in FIS world cup snowboarders and freestyle skiers: do real-life impacts exceed helmet testing standards? Br. J. Sports Med. 52, 32-40. doi: 10.1136/bjsports-2016-097086

Supej, M. (2010). 3D measurements of alpine skiing with an inertial sensor motion capture suit and GNSS RTK system. J. Sports Sic. 28, 759-769. doi: 10.1080/02640411003716934

Takeda, M., Miyamoto, N., Endo, T., Ohtonen, O., Lindinger, S., Linnamo, V., et al. (2019). Cross-Country skiing analysis and ski technique detection by high-precision kinematic global navigation satellite system. Sensors 19:4947. doi: $10.3390 / \mathrm{s} 19224947$

Conflict of Interest: The authors declare that the research was conducted in the absence of any commercial or financial relationships that could be construed as a potential conflict of interest.

Copyright (c) 2021 Spörri, Stöggl and Aminian. This is an open-access article distributed under the terms of the Creative Commons Attribution License (CC BY). The use, distribution or reproduction in other forums is permitted, provided the original author(s) and the copyright owner(s) are credited and that the original publication in this journal is cited, in accordance with accepted academic practice. No use, distribution or reproduction is permitted which does not comply with these terms. 